$\mathrm{Zu}$ diesen Schwächen treten darstellungstechnische Defizite. Die Arbeit weist, wenn der Rezensent richtig gezählt hat, 204 (!) Überschriften für Kapitel und Unterkapitel auf, das heißt, auf durchschnittlich jeder zweiten Seite beginnt der Autor mit einem neuen Thema. Eine Rückbindung empirischer Befunde an theoretische Fragestellungen ist damit ebenso wenig möglich wie die Entfaltung komplexer Argumentationslinien. Durchaus folgerichtig gleicht Eisele seine Befunde denn auch kaum mit Untersuchungen zu anderen Landesparlamenten ab oder ordnet sie in allgemeine Zusammenhänge ein.

$\mathrm{Zu}$ der im Wesentlichen auf Deskription zielenden Darstellung passt, dass der Autor seine Befunde gern in Tabellen, Übersichten und Diagrammen präsentiert. Viele davon sind erhellend und besitzen einen erkenntnisfördernden Mehrwert. Doch bleibt dem Rezensenten bei nicht wenigen der Zweck verborgen, den Eisele mit einer grafischen Darstellung von Informationen verbindet, die er dann noch einmal im Text präsentiert und erläutert. So mögen Tortendiagramme in Vorträgen nützlich sein, um - einfache - Verhältnisse zu illustrieren. In einem Buch scheint es jedoch überflüssig, das Verhältnis von zwei oder drei Prozentzahlen in eine Grafik zu übersetzen. So brauchen Leser wohl kein Tortendiagramm, um zu begreifen, wie viele Tagesordnungspunkte aus der Mitte des Landtags (65 Prozent), von der Regierung (32 Prozent) oder von Dritten (3 Prozent) initiiert wurden (S. 135). Das mag beckmesserisch klingen; doch stören die insgesamt 62 Grafiken, Übersichten und Tabellen nicht nur vielfach den Lesefluss, sondern in ihnen drückt sich auch das zentrale Anliegen Eiseles aus: Ihm geht es häufig mehr um die Beschreibung und Aufbereitung seiner Daten als um eine theoriegeleitete Analyse der Institution.

Insgesamt hat Eisele also eine in vielerlei Hinsicht bewundernswerte Fleißarbeit vorgelegt, deren Erkenntnispotential er aber leider nicht oder nur ansatzweise ausschöpft.

Werner Reutter

\title{
Der Bayerische Landtag: gute Aufbereitung für die politische Bildung
}

Haus der Bayerischen Geschichte (Hrsg.): Geschichte des Bayerischen Parlaments 1819-2003, CD-ROM, Augsburg 2005, € 12,-zzgl. Versandkosten.

Die CD-Rom zur Geschichte des Bayerischen Parlaments von 1819 bis 2003 wurde als Projekt des Hauses der Bayerischen Geschichte in Zusammenarbeit mit dem Landtagsamt des Bayerischen Landtags erstellt. Sie bietet eine Fülle an Text-, Daten-, Foto-/Film- und Tonmaterial zum Thema. Geordnet ist das Material über drei Zugänge. Der erste ist ein systematischer. Hier werden in acht Kapiteln Aufgaben, Organisation und Funktion des Bayerischen Landtags erörtert. Vorgestellt werden (1) Die Stellung des Landtags im politischen System, (2) Der Landtag als Gesetzgeber, (3) Die Abgeordneten, (4) Die Arbeit des Landtags, (5) Landtagspräsident und Landtagsamt, (6) Der Landtag und die Bürger, (7) Kompetenzverlust an den Bund und die EU und schließlich (8) Das Parlamentsgebäude. Der zweite Zugang ist historisch. Hier wird die Entwicklung des Bayerischen Parlaments seit 1819 (1. Landtag) vor dem Hintergrund der historischen Ausgangslage seit 1799 bis zum Landtag 1998 bis 2003 (14. Legislaturperiode) dargestellt. Dabei sind einerseits die relevanten Umstände und Ereignisse der bayerischen Parlamentsgeschichte chronologisch 
geordnet. Andererseits werden parallel dazu deren Verbindung und Einordnung in die Geschichte des Reichs und der Republik vorgenommen. Der dritte Bereich ist als Suchfunktion organisiert. Hier kann insbesondere nach Personen, alphabetisch, nach Ortsindex und Berufsindex gegliedert, sowie nach Landtagen (Landtage im Königreich, Landtage in der Weimarer Republik (plus ,gleichgeschalteter' Landtag ab 1933) und Landtage nach dem Zweiten Weltkrieg) mit den Rubriken Überblick, Gesetzgebung, Abgeordnete, Minister und Wahlergebnisse gesucht werden.

Die CD-Rom hat zwei Stärken und eine Schwäche. Ihre Stärke liegt erstens im für die politische Bildungsarbeit in Schule und Erwachsenenbildung ansprechend aufgearbeiteten Material. Ereignisse und Daten werden angemessen bebildert und durch kleine Film- oder Tonsequenzen nahe gebracht. Zweitens wird eine große Fülle für die Recherche ausgesprochen hilfreichen Datenmaterials geboten: eine Datenbank, die alle Abgeordneten des Bayerischen Landtags enthält, Details zu jeder einzelnen Sitzungsperiode des Landtags und andere relevante Dokumente. Statistisches Material wurde aufbereitet, und biographische Skizzen zu Funktion und Lebenslauf von Ministern wie Abgeordneten sind über die Zeit verfügbar. Die Schwäche zeigt sich im ersten systematischen Teil. Auch hier bekommt man überreich Informationen, Daten und Material geboten, die politikwissenschaftliche Analyse unterbleibt aber durchweg.

Wer sich über den Bayerischen Landtag von 1819 bis 2003 informieren möchte, wer Informationen, Daten, Material und Medien zum Thema sucht, wird hier ausgesprochen gut und umfassend bedient. Wer eine kritische Diskussion zu Funktion und Entwicklung des Parlamentarismus (auf Landesebene) sucht, die anspruchsvolle politische Bildung auch bieten sollte, bleibt auf die entsprechende Fachliteratur verwiesen.

Günter Rieger

\section{Europas Verfassungen - in Vielfalt geeint: beeindruckende Edition}

Gosewinkel, Dieter und Johannes Masing (Hrsg.): Die Verfassungen in Europa 1789 - 1949. Eine wissenschaftliche Textedition, Verlag C. H. Beck, München 2006, 2116 Seiten, € 268,-.

Europa gelingt gemeinsam - dies war das Motto der deutschen EU-Ratspräsidentschaft im Jahr 2007, und dieser Grundsatz prägt auch das werdende europäische Verfassungsrecht. Sowohl die Grundrechtecharta (Präambel) als auch der Verfassungsvertrag (Art. I-9 Abs. 3) nehmen ausdrücklich auf die ,gemeinsamen Verfassungstraditionen“ beziehungsweise die „gemeinsamen Verfassungsüberlieferungen der Mitgliedstaaten“ Bezug. Was das genau ist, woraus sich diese Traditionen und Überlieferungen speisen, war lange Zeit wenig deutlich. Jetzt sind die nationalen Wurzeln des europäischen Verfassungsrechts erstmals umfassend freigelegt: Dieter Gosewinkel und Johannes Masing haben in jahrelanger, mühevoller Kleinarbeit sämtliche Verfassungen der europäischen Staaten zusammengetragen, übersetzt und veröffentlichen sie nun erstmals vollständig und in deutscher Sprache.

Der zeitliche Rahmen der Edition reicht von 1789 bis 1949. Das Jahr der Französischen Revolution ist der Auftakt der modernen europäischen Verfassungsentwicklung, das Jahr 1949 verstehen die Herausgeber als Abschluss der Wiederherstellung der Verfassungsstaaten 